\title{
Immune-mediated Neuropathy with Anti-disialosyl IgM Antibodies in Diffuse Large B-cell Lymphoma: A Case Report and Literature Review
}

\author{
Gen Shihashi ${ }^{1}$, Takuya Yagi ${ }^{1}$, Shigeaki Suzuki ${ }^{1}$, Morinobu Seki ${ }^{1}$, Sumiko Kohashi ${ }^{2}$, \\ Tomoki Ueda ${ }^{2}$, Kaori Kameyama ${ }^{3}$, Susumu Kusunoki ${ }^{4}$, Hideaki Nakajima ${ }^{2}$, \\ Shinichiro Okamoto ${ }^{2}$ and Norihiro Suzuki ${ }^{1}$
}

\begin{abstract}
A 36-year-old man with diffuse large B-cell lymphoma presented with polyneuropathy, and the diagnostic work-up revealed the presence of IgM antibodies against gangliosides with disialosyl residues (GD1b, GD3). He was treated with rituximab, cyclophosphamide, doxorubicin, vincristine, and prednisone and received high-dose intravenous immunoglobulin for the treatment of neuropathy. After initiating the treatments, the patient's neurological impairment improved dramatically. He currently remains in complete remission without a flare-up of the polyneuropathy. Based upon our experience and other case reports of lymphoma with immune-mediated neuropathy caused by anti-disialosyl ganglioside IgM antibodies, we conclude that determining the anti-ganglioside antibody profile and beginning early treatment should be considered promptly for patients with malignant lymphoma who develop polyneuropathy.
\end{abstract}

Key words: Guillain-Barré syndrome, malignant lymphoma, immune-mediated neuropathy, anti-ganglioside antibody, paraneoplastic syndrome, diffuse large B-cell lymphoma

(Intern Med 54: 1647-1651, 2015)

(DOI: 10.2169/internalmedicine.54.4451)

\section{Introduction}

Peripheral neuropathy occasionally occurs in patients with malignant lymphoma. Various causes include direct tumor infiltration, neurotoxicity from chemotherapy, metabolic processes, infections, and paraneoplastic mechanisms (1). Paraneoplastic sensorimotor neuropathy, including GuillainBarré syndrome (GBS) and chronic inflammatory demyelinating polyneuropathy (CIDP), is infrequently observed in patients with lymphoma (2). However, immune-mediated neuropathy with anti-ganglioside antibodies is rare in this population (3-7). We herein report a patient with diffuse large B-cell lymphoma who also developed immunemediated neuropathy with anti-ganglioside antibodies. We also provide a short literature review in order to elucidate the clinical characteristics of patients with lymphoma and immune-mediated neuropathies with IgM antibodies against disialosyl gangliosides.

\section{Case Report}

A 36-year-old Japanese man had occasional bright red blood in his stools. Colonoscopy identified an ileocecal tumor, and the biopsy findings of the tumor confirmed the diagnosis of diffuse large B-cell lymphoma (Fig. 1A, B). He was referred to our hospital for further evaluation and treatment. The work-up at our hospital with [18F]-fluorodeoxyglucose positron emission tomography scans demonstrated a fluorodeoxyglucose uptake in the mass at the vesiculo-rectal

\footnotetext{
${ }^{1}$ Department of Neurology, Keio University School of Medicine, Japan, ${ }^{2}$ Division of Hematology, Department of Medicine, Keio University School of Medicine, Japan, ${ }^{3}$ Department of Pathology, Keio University School of Medicine, Japan and ${ }^{4}$ Department of Neurology, Kinki University School of Medicine, Japan

Received for publication November 3, 2014; Accepted for publication January 14, 2015

Correspondence to Dr. Takuya Yagi, takuyagi@ 2006.jukuin.keio.ac.jp
} 

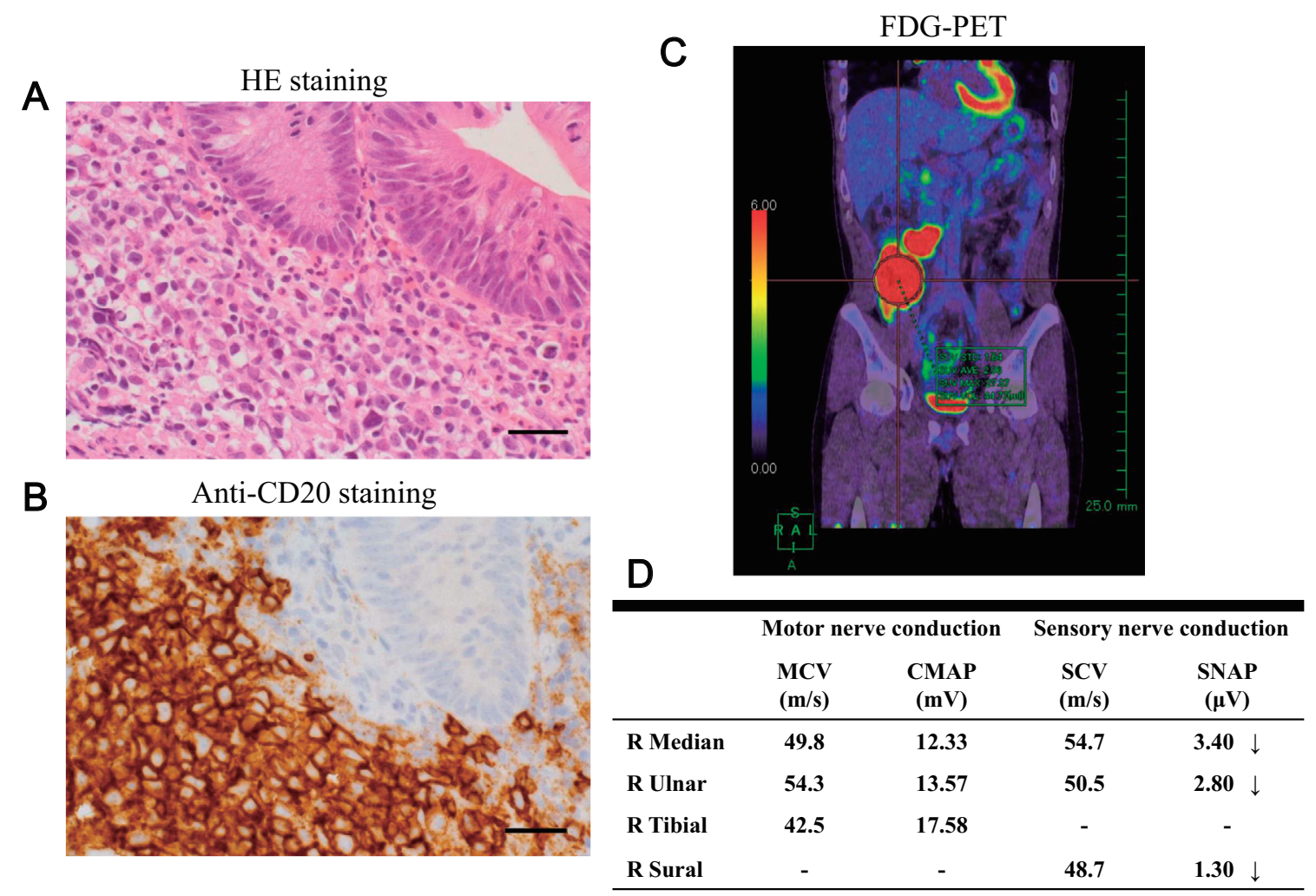

Figure 1. (A-C) The biopsy and a $\left[{ }^{18} \mathrm{~F}\right]$-fluorodeoxyglucose positron emission tomography (FDGPET) image findings. The biopsy findings of the ileocecal tumor showed large atypical cells with Hematoxylin and Eosin staining (A) and CD20-positive cells with immunohistological staining (B). A representative image of abdominal FDG-PET showed increased cellular activity in the ileocecal tumor (C). (D) Nerve conduction studies performed at Day 13. The compound muscle action potentials from wrist stimulation for the right median and ulnar nerves and from ankle stimulation for the right tibial nerve are shown. The sensory nerve conduction velocities and sensory nerve action potentials were measured orthodromically at distances of $150 \mathrm{~mm}, 110 \mathrm{~mm}$, and $150 \mathrm{~mm}$ between the stimulating and recording electrodes in the right median nerve, right ulnar nerve and right sural nerve, respectively. The scale bar indicates $50 \mu \mathrm{m}$.

fossa and an ileocecal lesion that included regional lymph nodes (Fig. 1C). Two weeks before admission to our hospital, the patient started complaining of dysesthesia in the distal extremities, and the cerebrospinal fluid (CSF) analysis at that time revealed albuminocytologic dissociation (total protein $98 \mathrm{mg} / \mathrm{dL}$, cells $0 / \mu \mathrm{L}$ ). At admission to our hospital (Day 0) (Fig. 2), he still complained of a mild sensory disturbance, but neither a motor deficit nor ataxia was detected on the neurological examination. The deep tendon reflexes were almost normal, and the findings from a nerve conduction study were within the normal limits.

Rituximab, cyclophosphamide, doxorubicin, vincristine, and prednisone therapy (R-CHOP) was initiated on Day 6. A few days after the initiation of R-CHOP, the patient's neurological symptoms gradually worsened. Painful paresthesia with a stocking-glove distribution appeared. A decreased sensation of all modalities in all four extremities, limb ataxia, and decreased tendon reflexes in the extremities were observed. Romberg's sign was also present. He also developed a mild motor deficit as determined by the Modified Medical Research Council's manual muscle testing
(MMT) scores of 4 in all limbs, although his cranial nerve functions and autonomic functions remained unaffected. The laboratory work-ups demonstrated that the serum $\operatorname{IgM}$ concentration was within normal limits $(124 \mathrm{mg} / \mathrm{dL})$, and no monoclonal $\operatorname{IgM}$ paraprotein was detected with immunoelectrophoresis. The total protein level in the CSF was significantly elevated $(321 \mathrm{mg} / \mathrm{dL})$ without elevation of the cell count $(0 / \mu \mathrm{L})$. No lymphoma cells were detected in the bone marrow or CSF. Nerve conduction studies, which were performed at Day 13, showed decreased sensory nerve action potentials, while the sensory nerve conduction velocities and motor conduction studies were almost within normal ranges, which were consistent with sensory dominant axonal polyneuropathy (Fig. 1D). An enzyme-linked immunosorbent assay (ELISA) revealed the presence of IgM antibodies against GD1b and GD3 in the serum. Other paraneoplastic anti-neuronal antibodies, including anti-Hu, anti-Ri, anti-Yo, anti-CV2, anti-Tr, and anti-Ma2, were absent. Based on the clinical features, the patient was diagnosed with immunemediated neuropathy with anti-disialosyl IgM antibodies. He underwent a 5-day course of high-dose intravenous immuno- 


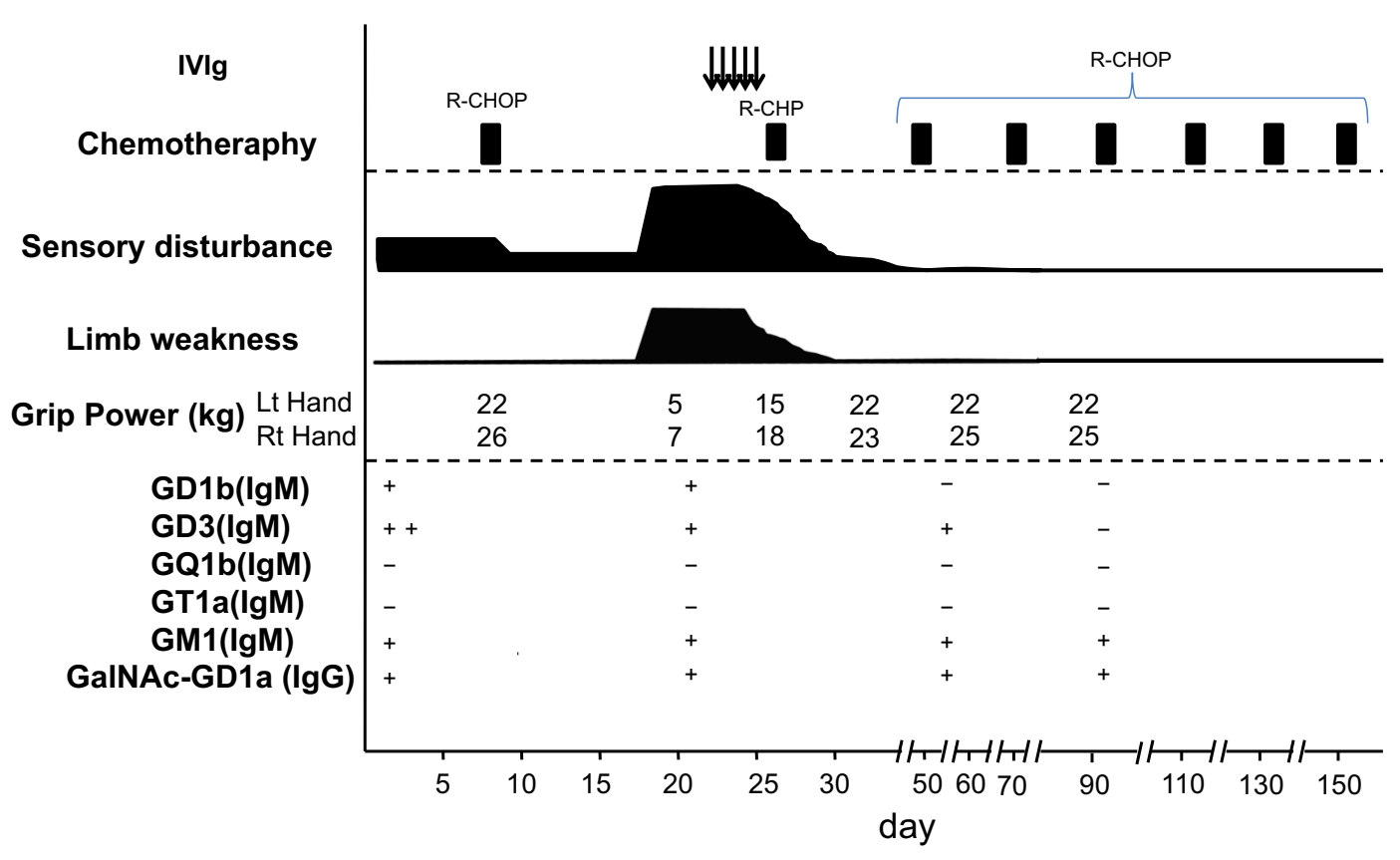

Figure 2. Clinical course and the serial analysis of anti-ganglioside antibodies. Serum samples were collected on day 2 (pre-treatment), day 22, day 57, and day 92 (post-treatment). Arrows indicate IVIG. The activities of IgM anti-ganglioside antibodies are indicated as +++ (strong), ++ (moderate), + (weak), and - (negative). R-CHP shows that vincristine was omitted from the R-CHOP regimen.

globulin (IVIG) therapy (0.4 g/kg/day) (Days 22-26) and continued to receive R-CHOP therapy, but vincristine was omitted from the second course of chemotherapy to avoid further exacerbation of the peripheral neuropathy. After the IVIG therapy, his motor and sensory disturbances improved dramatically. The MMT score returned to 5 in all limbs, and his painful paresthesia and disturbance of sensations in all modalities disappeared. The patient was discharged in stable condition (Day 43). IgM antibodies against disialosyl residues in the serum became undetectable on Day 92. He received a third course of R-CHOP with a $50 \%$ dose reduction of vincristine and five additional courses of R-CHOP without any dose modification. Eight months after the onset, the patient remains in complete remission without recurrence of the neuropathy or vincristine-induced peripheral neuropathy.

\section{Discussion}

Peripheral nervous system abnormalities are one of the common neurological complications observed in patients with malignant lymphoma with an incidence of approximately 5\% (8). The causes of peripheral neuropathy observed in lymphoma include direct invasion of the lymphoma, side effects from the cancer treatment, metabolic imbalance, nerve compression, infection, and paraneoplastic syndrome. Among these, neurolymphomatosis, which is nerve infiltration by lymphoma cells, is the major cause of lymphoma-related neuropathy (2). Conversely, a relationship may exist between GBS and malignant lymphoma (9). However, immune-mediated neuropathies, such as GBS and
CIDP, occur less frequently in lymphoma patients, and reported cases of neuropathies with anti-ganglioside antibodies are rare. Thus, the role of anti-ganglioside antibodies in such cases remains to be elucidated.

We herein describe a patient with diffuse large B-cell lymphoma and immune-mediated polyneuropathy with IgM antibodies against the disialosyl residues who had a favorable outcome after early treatment with IVIG and chemotherapy. Since the first case report of antibody-mediated neuropathy with disialylated gangliosides in 1985 (10), the clinical features of this type of neuropathy have been well documented in the literature $(11,12)$. The clinical features of immune-mediated polyneuropathy with $\operatorname{IgM}$ antibodies against the disialosyl residues are typically marked sensory ataxia, areflexia, relatively preserved motor function (except for ophthalmoplegia), and a chronic or relapsing course. The induction of sensory ataxic neuropathy in rabbits sensitized with GD1b ganglioside suggests that the disialosyl epitope of GD1b may be the main target for such anti-disialosyl antibodies (13). In the clinical course of the present case, sensory disturbance occurred first, followed by ataxia. Although this patient also developed mild motor symptoms during exacerbations, sensory-dominant neuropathy was considered to be the clinical entity in this case. As neurological symptoms improved in this patient after treatment, $\operatorname{IgM}$ antibodies against disialosyl residues (GD1b, GD3) became undetectable in the serum. An analysis of other anti-ganglioside antibodies in this case showed that GM1 IgM and GalNAcGD1a IgG were positive, but no alteration in the antibody activity was observed for these antibodies throughout the entire clinical course. Thus, a non-specific reaction may have 
Table. Summary of Reported Cases of Immune-mediated Neuropathy Associated with IgM Antibodies against Disialosyl Gangliosides in Association with Malignant Lymphoma.

\begin{tabular}{|c|c|c|c|c|c|c|}
\hline $\begin{array}{c}\text { Case } \\
\text { (Reference) }\end{array}$ & $\begin{array}{l}\text { Age, } \\
\text { gender }\end{array}$ & Types of malignant lymphoma & Types of neuropathy & Anti-ganglioside Profile & Treatment & Clinical course \\
\hline 3 & $68, \mathrm{M}$ & Hairy Cell Leukemia lymphoma & $\begin{array}{l}\text { Acute motor dominant } \\
\text { polyneuropathy }\end{array}$ & $\begin{array}{l}\text { GM1 IgM (asialo form), } \\
\text { GD1b IgM }\end{array}$ & $\begin{array}{l}\text { Plasmapheresis, } \\
\text { Chemotherapy }\end{array}$ & Not described \\
\hline 4 & $76, \mathrm{~F}$ & $\begin{array}{l}\text { Rectal low-grade B-cell } \\
\text { lymphoma }\end{array}$ & $\begin{array}{l}\text { Subacute sensorimotor } \\
\text { polyneuropathy } \\
\text { (demyelinating) }\end{array}$ & GD1b IgM & IVIg, PSL & $\begin{array}{l}\text { Died } 2 \text { months from the } \\
\text { onset }\end{array}$ \\
\hline 5 & $52, \mathrm{M}$ & Diffuse large B-cell lymphoma & $\begin{array}{l}\text { Multifocal motor neuropathy } \\
\text { (demyelinating) }\end{array}$ & GM1 IgM, GD1b IgM & $\begin{array}{l}\text { IVIg, } \\
\text { Plasma exchange, } \\
\text { Chemotherapy }\end{array}$ & $\begin{array}{l}\text { Died } 4 \text { months from the } \\
\text { onset }\end{array}$ \\
\hline 6 & $63, \mathrm{M}$ & Diffuse large B-cell lymphoma & $\begin{array}{l}\text { Chronic sensorimotor } \\
\text { polyneuropathy }\end{array}$ & $\begin{array}{l}\text { GD3 IgM, GD2 IgM, } \\
\text { GD1b IgM, GT1b IgM, } \\
\text { GQ1b IgM, } \\
\text { fucosyl GD1b IgM, } \\
\text { GT1a IgM }\end{array}$ & $\begin{array}{l}\text { Plasmapheresis, } \\
\text { IVIg, PSL }\end{array}$ & $\begin{array}{l}\text { Died } 7 \text { years from the onset } \\
\text { after repeated remissions and } \\
\text { relapses }\end{array}$ \\
\hline 7 & $60, \mathrm{M}$ & Mantle cell lymphoma & $\begin{array}{l}\text { Chronic motor dominant } \\
\text { polyneuropathy } \\
\text { (demyelinating) }\end{array}$ & $\begin{array}{l}\text { GD1b IgM, GD3 IgM, } \\
\text { GT1b IgM, GQ1b IgM }\end{array}$ & $\begin{array}{l}\text { PSL, } \\
\text { Plasmapheresis, } \\
\text { Plasma exchange, } \\
\text { Chemotherapy }\end{array}$ & $\begin{array}{l}\text { Alive and neurologically } \\
\text { improved }\end{array}$ \\
\hline Present case & $36, \mathrm{M}$ & Diffuse large B-cell lymphoma & $\begin{array}{l}\text { Acute sensorimotor } \\
\text { polyneuropathy (axonal) }\end{array}$ & $\begin{array}{l}\text { GD1b IgM, GD3 IgM, } \\
\text { GM1 IgM, } \\
\text { GalNAc-GD1a IgG }\end{array}$ & $\begin{array}{l}\text { IVIg, } \\
\text { Chemotherapy }\end{array}$ & $\begin{array}{l}\text { Alive and neurologically } \\
\text { improved }\end{array}$ \\
\hline
\end{tabular}

IVIg: Intravenous Immunoglobulin, PSL: Predonisolone

been caused by natural antibodies, and these antibodies were unlikely to have contributed to the clinical features. Based on the clinical course and antibody results, we hypothesize that IgM antibodies against disialosyl residues (GD1b, GD3) were the main contributors to the pathogenesis in this case.

Because the clinical characteristics of lymphoma patients with neuropathy associated with anti-disialosyl IgM antibodies are unknown, we summarized the currently reported cases. The PubMed database was used to search for cases of malignant lymphoma with neuropathy related to antidisialosyl IgM antibodies. We identified five reported cases from this PubMed literature search, and their clinical characteristics, including that of the present case, are summarized in Table. Although relatively preserved motor function is a common feature of neuropathy related to anti-disialosyl IgM antibodies, all patients developed motor symptoms. Interestingly, the clinical courses varied widely in these patients. First, the onset of neurological symptoms differed among the patients. Neuropathy preceded the manifestations of malignant lymphoma in one case (6). In contrast, neuropathy occurred even after chemotherapy and at the time of recurrence of primary disease in another case (7). In the present case, neurological symptoms gradually occurred just after the diagnosis of malignant lymphoma, thus, chemotherapy and IVIG were initiated at almost the same time. Second, the classification of immune-mediated neuropathy as an acute pattern (GBS) or a chronic pattern (CIDP) also differed among the cases. Although one case had a CIDP-like course with repeated relapses and remissions over several years (6), the present case showed a GBS-like clinical course, which was a monophasic subacute illness with neu- rological deterioration and remission within 1 month. Third, the response to treatments and the prognosis also varied among the cases. Although three of the six patients who did not respond to the treatments died, the other patients, including the present patient, responded well to the treatments (7). As the long-term prognosis may be strongly influenced by the status of the malignant lymphoma, various factors (such as the type of lymphoma, clinical staging, and the patient age) may make an accurate prognosis difficult. The review of the literature in this report showed that the clinical features of neuropathy related to anti-disialosyl IgM antibodies in lymphoma patients may be unpredictable. The present case suggests that proper assessment may be important for early treatment and a favorable outcome. Thus, early serological screening for anti-ganglioside antibodies should be considered promptly in lymphoma patients who present with symptoms of peripheral neuropathy, because the detection of anti-ganglioside antibodies may lead to the appropriate diagnosis and early treatment.

Whether GBS is associated with cancer, including lymphoma, is debatable. Some reports have supported the possible correlation between GBS and cancer $(14,15)$, and it is hypothesized that the pathophysiology is due to antibodies derived from the molecular mimicry of antigens in lymphoma cells which may attack similar antigens in the peripheral nerve cells (1). Among the patients which were listed in Table, Noguchi et al. demonstrated that their patient's B cell lymphoma produced a monoclonal IgM autoantibody against glycolipids GM1 and GD1b, which caused multifocal motor neuropathy (5). Therefore, we speculated that anti-disialosyl IgM antibodies may be medi- 
ated by lymphoma in the present case. In regard to the treatments, there has been no randomized controlled trials for paraneoplastic neuropathy; thus, the clinical evidence is limited to small sequential or retrospective studies, case reports or expert opinions (16). Therefore, it is generally considered that the treatments should focus on tumor treatment and immunomodulation, mainly through IVIG therapy.

In conclusion, the present case demonstrated that in lymphoma, early treatment for immune-mediated neuropathy with IgM antibodies against the disialosyl residues resulted in a favorable outcome. Careful consideration is needed for the diagnosis of lymphoma patients who develop neurological symptoms, because the neurological manifestations in lymphoma patients have a wide differential diagnosis. In particular, peripheral neuropathy associated with lymphoma covers a broad range of causes, resulting in a difficult diagnosis.

The authors state that they have no Conflict of Interest (COI).

\section{Acknowledgement}

We are grateful to Dr. Keiko Tanaka and Dr. Yoko Kitagawa (Kanazawa Medical University) for their help in the serologic analyses of paraneoplastic anti-neuronal antibodies.

Gen Shihashi and Takuya Yagi contributed equally to this work.

\section{References}

1. Kelly JJ, Karcher DS. Lymphoma and peripheral neuropathy: a clinical review. Muscle Nerve 31: 301-313, 2005.

2. Tomita M, Koike H, Kawagashira Y, et al. Clinicopathological features of neuropathy associated with lymphoma. Brain 136: 25632578, 2013.

3. Sarmiento MA, Neme D, Fornari MC, Bengio RM. Guillain-Barré syndrome following 2-chlorodeoxyadenosine treatment for Hairy Cell Leukemia. Leuk Lymphoma 39: 657-659, 2000.
4. Marfia GA, Pachatz C, Terracciano C, et al. Subacute demyelinating polyneuropathy in B-cell lymphoma with $\operatorname{IgM}$ antibodies against glycolipid GD1b. Neurol Sci 26: 355-357, 2005.

5. Noguchi M, Mori K, Yamazaki S, Suda K, Sato N, Oshimi K. Multifocal motor neuropathy caused by a B-cell lymphoma producing a monoclonal $\operatorname{IgM}$ autoantibody against peripheral nerve myelin glycolipids GM1 and GD1b. Br J Haematol 123: 600-605, 2003.

6. Kobayashi M, Kato K, Funakoshi K, Watanabe S, Toyoshima I. Neuropathology of paraneoplastic neuropathy with anti-disialosyl antibody. Muscle Nerve 32: 216-222, 2005.

7. Mori A, Ueno Y, Kuroki T, et al. Motor-dominant polyneuropathy due to IgM monoclonal antibody against disialosyl gangliosides in a patient with mantle cell lymphoma. J Neurol Sci 337: 215-218, 2014.

8. Hughes RA, Britton T, Richards M. Effects of lymphoma on the peripheral nervous system. J R Soc Med 87: 526-530, 1994.

9. Koike H, Tanaka F, Sobue G. Paraneoplastic neuropathy: wideranging clinicopathological manifestations. Curr Opin Neurol 24: 504-510, 2011.

10. Ilyas AA, Quarles RH, Dalakas MC, Fishman PH, Brady RO. Monoclonal IgM in a patient with paraproteinemic polyneuropathy binds to gangliosides containing disialosyl groups. Ann Neurol 18: 655-659, 1985.

11. Yuki N, Uncini A. Acute and chronic ataxic neuropathies with disialosyl antibodies: a continuous clinical spectrum and a common pathophysiological mechanism. Muscle Nerve 49: 629-635, 2014.

12. Willison HJ, O'Leary CP, Veitch $\mathrm{J}$, et al. The clinical and laboratory features of chronic sensory ataxic neuropathy with antidisialosyl IgM antibodies. Brain 124: 1968-1977, 2001.

13. Kusunoki S, Shimizu J, Chiba A, Ugawa Y, Hitoshi S, Kanazawa I. Experimental sensory neuropathy induced by sensitization with ganglioside GD1b. Ann Neurol 39: 424-431, 1996.

14. Vigliani MC, Magistrello M, Polo P, Mutani R, Chio A. Risk of cancer in patients with Guillain-Barré syndrome (GBS). A population-based study. J Neurol 251: 321-326, 2004.

15. Antoine JC, Camdessanche JP, Ferraud K, Caudie C. Antiganglioside antibodies in paraneoplastic peripheral neuropathies. J Neurol Neurosurg Psychiatry 75: 1765-1767, 2004.

16. Giometto B, Vitaliani R, Lindeck-Pozza E, Grisold W, Vedeler C. Treatment for paraneoplastic neuropathies. Cochrane Database Syst Rev 12: CD007625, 2012.

(C) 2015 The Japanese Society of Internal Medicine http://www.naika.or.jp/imonline/index.html 